\title{
Magnetoelastic effects and the magnetic phase diagram of multiferroic $\mathrm{DyMn}_{2} \mathrm{O}_{5}$
}

\author{
C. R. dela Cruz, ${ }^{1}$ B. Lorenz, ${ }^{1}$ Y. Y. Sun, ${ }^{1}$ C. W. Chu, ${ }^{1,2,3}$ S. Park, ${ }^{4}$ and S.-W. Cheong ${ }^{4}$ \\ ${ }^{1}$ Department of Physics and TCSUH, University of Houston, Houston, Texas 77204-5002, USA \\ ${ }^{2}$ Lawrence Berkeley National Laboratory, 1 Cyclotron Road, Berkeley, California 94720, USA \\ ${ }^{3}$ Hong Kong University of Science and Technology, Hong Kong, China \\ ${ }^{4}$ Department of Physics \& Astronomy and Rutgers Center for Emergent Materials, Rutgers University,
} Piscataway, New Jersey 08854, USA

(Received 27 July 2006; published 7 November 2006)

\begin{abstract}
The magnetoelastic coupling in multiferroic DyMn${ }_{2} \mathrm{O}_{5}$ is investigated by magnetostriction measurements along the three crystallographic orientations. Strong lattice anomalies as a function of the magnetic field are detected at the low temperature magnetic and ferroelectric phase transitions. The sign and magnitude of the magnetostrictive coefficient as well as the lattice anomalies at the transitions are correlated with the Dy moment order and with the sharp changes of the dielectric constant and the ferroelectric polarization. With the magnetic field applied along the $c$ axis a high-field phase has been detected the magnetic structure of which has yet to be explored.
\end{abstract}

DOI: 10.1103/PhysRevB.74.180402

PACS number(s): 75.25.+z, 75.30.Kz, 75.80.+q, 77.80.-e

Among the multiferroic materials displaying the coexistence of magnetic and ferroelectric (FE) orders the rare earth manganites $R \mathrm{Mn}_{2} \mathrm{O}_{5}(R=$ rare earth, $\mathrm{Y})$, have attracted attention because of a wealth of fundamental physical phenomena observed in these compounds. Incommensurate magnetic orders, spin frustration, lock-in transitions, and ferroelectricity have been detected with decreasing temperature and give rise to a cascade of phase transitions. ${ }^{1}$ The physical origin of this phase complexity lies in the partially competing interactions between the $\mathrm{Mn}^{4+} / \mathrm{Mn}^{3+}$ spins, the rare earth magnetic moments, and the lattice. ${ }^{2}$ Geometric magnetic frustration among the $\mathrm{Mn}^{3+}-\mathrm{Mn}^{4+}$ spins leads to a ground state degeneracy of the magnetic states that can be lifted by a distortion of the lattice (magnetic Jahn-Teller effect). This is believed to be the origin of ferroelectricity in $R \mathrm{Mn}_{2} \mathrm{O}_{5}$ compounds. ${ }^{2-4}$ Strong spin-lattice coupling is needed to explain the ionic displacements in the FE state. The experimental proof of sizable lattice anomalies at the FE transitions in $R \mathrm{Mn}_{2} \mathrm{O}_{5}$ was given recently by thermal expansion experiments in zero magnetic field. ${ }^{4}$ However, the effect of an external magnetic field on the lattice of $R \mathrm{Mn}_{2} \mathrm{O}_{5}$ has not been investigated yet. The field couples to the magnetic order resulting in fieldinduced spin reorientations and magnetic phase transitions ${ }^{5}$ which, in turn, should affect the lattice via the spin-lattice interaction. The signature and the nature of the magnetoelastic effect provides essential information about the intrinsic magnetoelastic and magnetoelectric interactions. We have therefore investigated the magnetoelastic strain on the lattice of DyMn $\mathrm{Mn}_{5}$ and found large changes of the lattice parameters as a function of the magnetic field and abrupt striction effects at phase boundaries.

DyMn $\mathrm{O}_{5}$ exhibits a complex magnetic phase diagram. ${ }^{5,6}$ Large effects of an $a$-axis magnetic field on the dielectric constant $^{7}$ and the FE polarization ${ }^{6}$ have been reported. Upon decreasing temperature $T$ the sequence of phase transitions is similar for most $R \mathrm{Mn}_{2} \mathrm{O}_{5}$. AFM order develops at $T_{N 1}$ $=43 \mathrm{~K}$ with an incommensurate (IC) magnetic modulation described by $\vec{q}=(0.5+\beta, 0,0.25+\delta)$ (where $\beta, \delta<0$ indicate the deviations from commensurability) followed by a lock-in transition at $T_{C 1}=39 \mathrm{~K}$ into a commensurate $\mathrm{FE}$ phase with $\vec{q}=(0.5,0,0.25)$. At the low- $T$ side a transition into a reentrant IC magnetic phase with modulation vector $\vec{q}$ $\approx(0.48,0,0.3)$ was reported for all $R \mathrm{Mn}_{2} \mathrm{O}_{5}\left(T_{C 3}=6.5 \mathrm{~K}\right.$ in DyMn $\mathrm{Mn}_{5}$ ). DyMn $\mathrm{O}_{5}$ experiences two more phase changes in between $T_{C 1}$ and $T_{C 3}$, a spin reorientation at $T_{N 2}=27 \mathrm{~K}$ and a lock-in transition accompanied by another spin reorientation at $T_{C 2}=13 \mathrm{~K} .{ }^{5}$ Figure 1 shows the dielectric constant upon heating with the phase transition temperatures and the different phases labeled as used throughout this communication. All five phase transitions are clearly visible as distinct anomalies of the dielectric constant, the FE polarization, ${ }^{6}$ the heat capacity, and the lattice parameters. ${ }^{4}$ The strongest lattice anomalies have been observed at $T_{C 3}$ where the Dy moments undergo the AFM odering with $\vec{q}_{\text {Dy }}$ $=(0.5,0,0)$ and the FE polarization drops to a small value close to zero. This reentrant paraelectric phase and its stability with respect to magnetic fields is of particular interest.

Single crystals of $\mathrm{DyMn}_{2} \mathrm{O}_{5}$ have been grown as described elsewhere. ${ }^{7}$ The longitudinal magnetostriction measurements were conducted along the three crystallographic orientations in an Oxford variable temperature cryostat with a superconducting magnet up to $14 \mathrm{~T}$ employing a highprecision capacitance dilatometer. ${ }^{4}$ Complimentary measure-

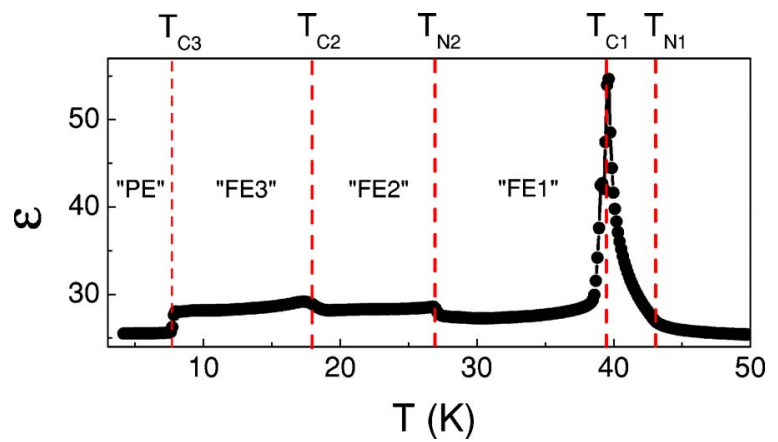

FIG. 1. (Color online) Dielectric constant of $\mathrm{DyMn}_{2} \mathrm{O}_{5}$ with all anomalies indicating transitions between different magnetic and $\mathrm{FE}$ structures. Data shown are for increasing $T$. 

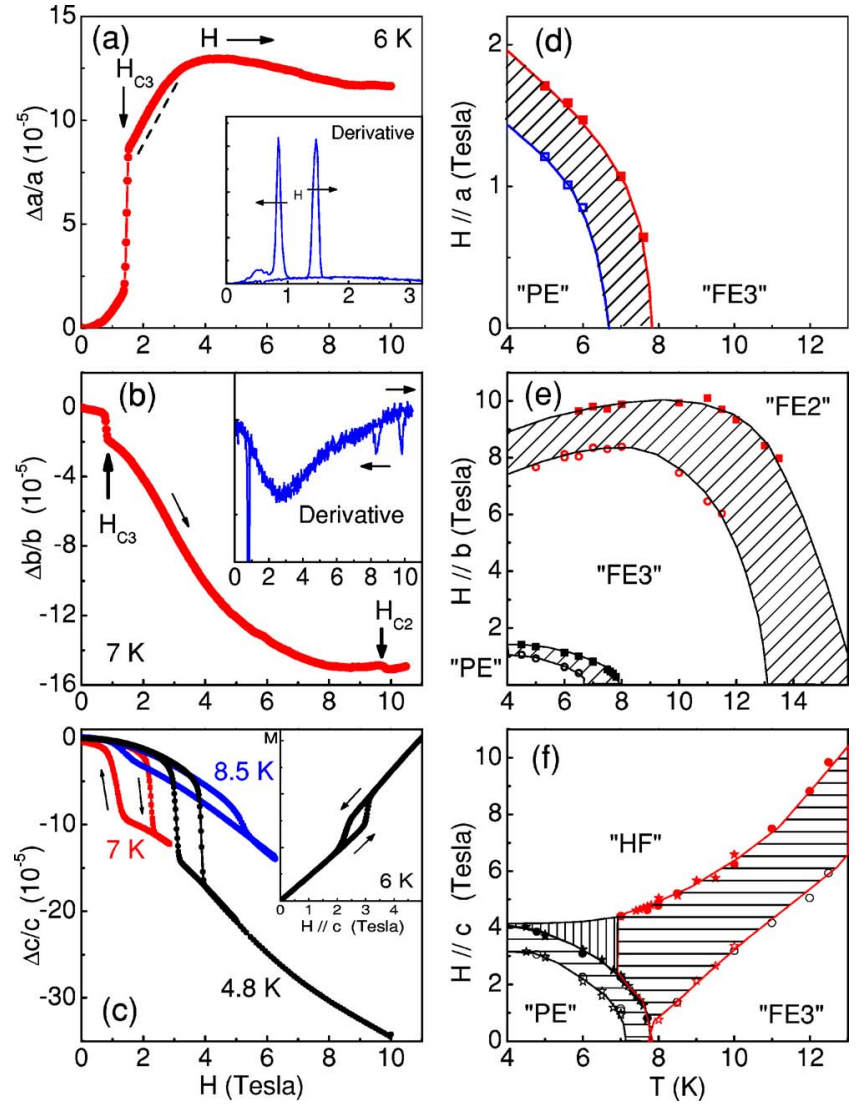

FIG. 2. (Color online) (a)-(c) Longitudinal magnetostriction along the $a, b$, and $c$ axes of $\mathrm{DyMn}_{2} \mathrm{O}_{5}$ [Data in the main panels of (a) and (b) are shown for increasing field only, the insets show the derivative for both field directions.] The inset to (c), $c$-axis magnetization vs field, shows the metamagnetic transition at the phase boundary $\mathrm{PE} \rightarrow \mathrm{HF}$. (d)-(f) Derived low-temperature phase diagrams. The regions of field hysteresis are indicated by dashed lines.

ments of the $b$-axis dielectric constant in magnetic field have been conducted to correlate the observed field-induced lattice anomalies with the dielectric properties and the known phase transitions. The dielectric constant was derived from the capacitance of a thin platelet with silver electrodes measured by the HP4285A LCZ meter at $1 \mathrm{MHz}$.

Figure 2(a) shows $\Delta a / a$ increasing with the magnetic field $H_{a}$ at $6 \mathrm{~K}$. The sharp step at $H_{C 3}=1.44 \mathrm{~T}$ indicates the first order phase transition from the PE phase to the FE3 phase with a field hysteresis of $0.85 \mathrm{~T}$. The inset of Fig. 2(a) shows the derivative (magnetostrictive coefficient) and the sharp peaklike anomalies with increasing and decreasing field at $H_{C 3}$. The field hysteresis is in accordance with the temperature hysteresis at $T_{C 3}$ observed in various physical quantities. The phase boundary between the PE and FE3 phases [Fig. 2(d)] is derived from the lattice anomalies at different temperatures. The dashed area designates the hysteretic region in the phase diagram. The phase boundary is in good agreement with the phase diagram derived from polarization measurements ${ }^{6}$ and from neutron scattering experiments. ${ }^{5}$ No additional lattice anomalies could be observed up to $13 \mathrm{~T}$. The previously observed field-induced transitions from FE3 to FE2 (Ref. 6) or into more complex structures $^{5}$ are apparently not accompanied by sizable changes of the $a$-axis length. However, above $H_{C 3}$ we can identify a linear magnetoelastic region extending to about $3 \mathrm{~T}$ [dashed line in Fig. 2(a)], similar to the field-induced linear magnetoelastic effect discovered in $\mathrm{TbMnO}_{3} .{ }^{8}$ In this field range the growth of a ferromagnetic (FM) moment and a change of the polarization extending to about $4 \mathrm{~T}$ [the maximum of $a(H)]$ has been observed. ${ }^{5}$ It appears conceivable that the linear magnetoelastic region and the following maximum of $a(H)$ are related to the commensurate FM and the incommensurate FM phases with a supposedly canted spin structure suggested by neutron scattering experiments.

The expansion of the $a$ axis with the field has its origin in the release of the strong AFM interaction of the Dy. The magnetic structure of the PE phase is dominated by the AFM order of the Dy moments along $a, \vec{q}_{\mathrm{Dy}}=(0.5,0,0)$. The magnetic system can gain exchange energy by reducing the distance of the ordered Dy moments along $a$ resulting in the large and sudden contraction of the $a$ axis below $T_{C 3}{ }^{4}$ The external field $H_{a}$ reduces the AFM Dy order along this axis and partially releases the lattice strain causing the increase of $a$ at small $H_{a}$. At the critical field $H_{C 3}$ neutron scattering experiments have indicated a reorientation of the Dy moments and a sudden reduction of their mutual correlations. This results in the sharp increase of $a$ at $H_{C 3}$ [Fig. 2(a)]. Our results provide clear evidence for the strong coupling of the Dy moments to the lattice.

The length of the $b$ axis decreases with increasing $H_{b}$ [Fig. 2(b)]. The transition from PE to FE3 is clearly seen as a sharp drop of $b$ at $H_{C 3}$. At higher fields a second steplike decrease of $b$ at $H_{C 2}$ indicates the field-induced transition into the FE2 phase. Both phase transitions exhibit a distinct field hysteresis in accordance with the temperature hysteresis at $T_{C 3}$ and $T_{C 2}$ observed at zero field. The data shown in Fig. 2(b) at $7 \mathrm{~K}$ fall into the temperature hysteretic region close to $T_{C 3}$ and the reverse transition from FE3 to PE does not take place in decreasing field. The phase sequence at $7 \mathrm{~K}$ with cycling the magnetic field from 0 to $11 \mathrm{~T}$ and back is therefore $\mathrm{PE}\left(H_{b}=0\right) \Rightarrow \mathrm{FE} 3\left(H_{b}>1 \mathrm{~T}\right) \Rightarrow \operatorname{FE} 2\left(H_{b}=11 \mathrm{~T}\right)$ $\Rightarrow \mathrm{FE} 3\left(H_{b}=0\right)$. Above $8 \mathrm{~K}$ the low-field anomaly of $b$ disappears and the high-field drop of $b(H)$ can be traced to $T>15 \mathrm{~K}$. The phase diagram is shown in Fig. 2(e). The shaded areas designate the hysteretic regions. Both phase boundaries match the zero-field transition temperatures $T_{C 3}$ and $T_{C 2}$. The stability of the FE3 phase extends to far higher fields as in the case of $H \| a^{5,6}$ The opposite sign of the magnetostriction along $b$ as compared to $a$ is consistent with the anisotropy of the thermal expansion anomalies observed at $T_{C 3} .{ }^{4}$ The low-temperature PE phase shows a sizable reduction of the $a$-axis length but a large increase of $b$ and $c$. The shrinkage of $a$ is driven by the Dy-moment order along $a$ as discussed above. The expansion of $b$ and $c$ below $T_{C 3}$ is then a consequence of the elastic forces of the lattice. The external magnetic field applied along all directions destabilizes the PE phase and releases the magnetostrictive strain. This results in the increase of $a$ but a decrease of $b$ and $c$ with the applied field.

The $c$ axis of $\mathrm{DyMn}_{2} \mathrm{O}_{5}$ is the hard magnetic axis ${ }^{7}$ and the magnetic phase diagram for $H_{c}$ has not been investigated yet. 
The magnetostriction measurements along $c$ reveal a more complex phase diagram as compared to the $a$ - and $b$-axis fields. At low $T$ a sharp drop of $c(H)$ at about $4 \mathrm{~T}$ indicates a phase transition with a field hysteresis of up to $1 \mathrm{~T}$. Magnetostriction data at different temperatures are shown in Fig. 2(c). The phase boundary of the PE phase shifts to lower fields with increasing temperature and vanishes above $7.8 \mathrm{~K}$. At higher $T$, however, another magnetostrictive anomaly above $4 \mathrm{~T}$ indicates a phase transition into a high-field (HF) phase. The critical field of this transition increases with increasing $T$ [Fig. 2(f)]. The transition FE3 $\Leftrightarrow$ HF exhibits a much larger field hysteresis of up to $3.5 \mathrm{~T}$ [as can be seen from the $8.5 \mathrm{~K}$ data of Fig. 2(c) and the phase diagram of Fig. 2(f)]. The magnetic structure of the HF phase is yet to be explored. It appears conceivable that the magnetic field realigns the Dy moments resulting in a spin-flop transition similar to that observed in $\mathrm{GdMnO}_{3}{ }^{9}$ The $c$-axis magnetization [inset to Fig. 2(c)] reveals a metamagnetic transition at this phase boundary that is consistent with a spin-flop scenario. The critical field indicating the entrance into the HF phase depends on the starting phase at zero field. For temperatures below $T_{C 3}$ the transition $\mathrm{PE} \Rightarrow \mathrm{HF}$ happens below $4 \mathrm{~T}$ and is associated with a larger step of $c(H)$ [data at 4.8 and $7 \mathrm{~K}$ in Fig. 2(c)], whereas the transition from FE3 to HF proceeds above $4 \mathrm{~T}$ with a significantly smaller change of the $c$ axis upon increasing field. Due to the wide hysteresis of the transitions there is a large region in the phase diagram (indicated by horizontal dashed lines) in which a strong $(T, H)$-history dependence of the final state can be observed. An example is given in Fig. 3(a). At a temperature of $7 \mathrm{~K}$ both phases PE and FE3 can be stabilized depending on approaching this temperature from below or above, respectively. Cooling to $7 \mathrm{~K}$ preserves the system in the FE3 phase. Increasing the magnetic field from zero to above $5 \mathrm{~T}$ transforms the system into the HF phase. The subsequent release of the field causes a phase transition from the HF phase into the PE phase at about $1 \mathrm{~T}$. The $c$-axis lattice parameter shows the sharp drop at the $\mathrm{FE} 3 \Rightarrow \mathrm{HF}$ transition with increasing $H$ and a large increase at the $\mathrm{HF} \Rightarrow \mathrm{PE}$ transition with the release of $H$ to zero. After the field cycle, in the final state, $c$ is larger than in the initial state. The difference of $\Delta c / c \approx 6 \times 10^{-5}$ is in perfect agreement with the lattice strain across $T_{C 3}$ obtained from zero-field thermal expansion experiments. ${ }^{4}$ Due to the thermal hysteresis region around $7 \mathrm{~K}$ at $H=0$ the external field can "switch" the magnetic and FE states and the $c$-axis length as indicated in Fig. 3(a).

The phase diagram of Fig. 2(f) has been verified by measurements of the dielectric constant $\varepsilon(T, H)$ in magnetic fields up to $7 \mathrm{~T}$. The various phase transitions between the PE, FE3, and HF phases are clearly detected in sharp changes of $\varepsilon$ upon increasing and decreasing fields. Some examples of $\varepsilon(H)$ are shown in Figs. 3(b)-3(d). The phase boundaries derived from dielectric measurements agree perfectly with the ones from magnetostriction data and they are included as stars in Fig. 2(f). Moreover, the irreversible phase change $\mathrm{FE} 3 \Rightarrow \mathrm{HF} \Rightarrow \mathrm{PE}$ while cycling $H$ at $7 \mathrm{~K}$ is also verified from the dielectric data [included as stars in Fig. $3(\mathrm{a})$ ]. There is a small region in the phase diagram between 7 and $7.8 \mathrm{~K}$ where the increase of $H_{c}$, starting from the low-

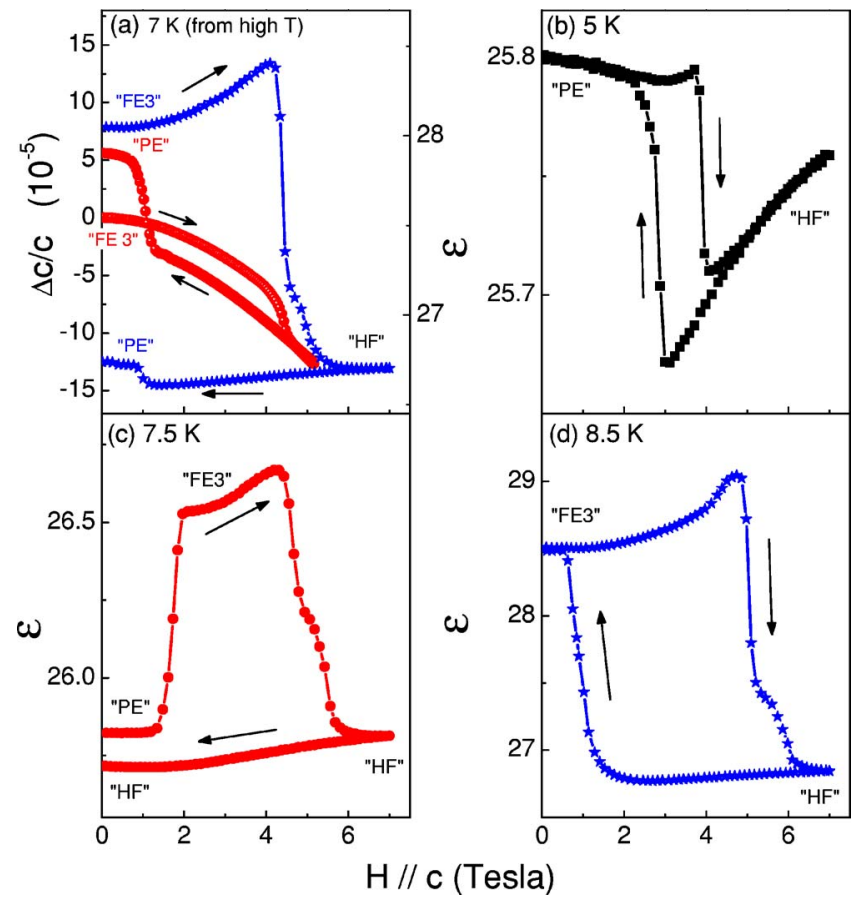

FIG. 3. (Color online) (a) Magnetostriction of the $c$ axis (circles, left scale) and dielectric constant (stars, right scale) at $7 \mathrm{~K}$ showing the irreversible change from FE3 to PE induced by the magnetic field cycle. (b)-(d) Dielectric constant at three characteristic temperatures showing sharp anomalies at all magnetic transitions between the PE, FE3, and HF phases. The directions of field change are indicated by arrows.

temperature PE phase, results in two subsequent phase transitions, first into the FE3 phase and then into the HF phase. Figure 3(c) shows $\varepsilon(H)$ at $7.5 \mathrm{~K}$. With increasing field the sharp increase of $\varepsilon$ is due to the transition $\mathrm{PE} \Rightarrow \mathrm{FE} 3$, whereas the sudden drop at higher field signals the second transition $\mathrm{FE} 3 \Rightarrow \mathrm{HF}$. Upon decreasing field the large hysteresis prevents any further phase change [Fig. 3(c)].

The major phase boundaries of Fig. 2(f) have been derived from magnetostriction and dielectric constant measurements at constant temperature with changing magnetic field. In order to uniquely identify the stability regions of the different phases, temperature dependent measurements of $\varepsilon$ at constant field have been conducted. The major phase boundaries were found in agreement with the field dependent measurements, however, the data have revealed an additional hysteretic region below $7 \mathrm{~K}$ close to $4 \mathrm{~T}$. This area is marked by vertical dashes in Fig. 2(f). With the field and temperature dependent measurements of $c(T, H)$ and $\varepsilon(T, H)$ we can identify the thermodynamically stable single phase regions of the phase diagram [shown by the blank areas in Fig. 2(f)]. In between these stable phase regions two or more phases can coexist and the magnetic and FE states of $\mathrm{DyMn}_{2} \mathrm{O}_{5}$ are history dependent.

The HF phase detected in magnetic fields along the $c$ axis and the strong lattice anomalies across the phase boundaries indicate that external fields have a significant effect not only if they are aligned with the axis of easy magnetization $a$ but also along the hard magnetic axis $c$, contributing to an in- 
creased phase complexity in $\mathrm{DyMn}_{2} \mathrm{O}_{5}$. This is not surprising since the magnetic modulation of the Mn and Dy order has an important component along the $c$ axis resulting in a spiral order of moments along $c$ which, depending on the temperature range, can be commensurate $\left(q_{z}=0.25\right)$ or incommensurate $\left(q_{z}=0.25+\delta\right){ }^{5}$ Recent investigations of the magnetic phase diagram of $\mathrm{DyMn}_{2} \mathrm{O}_{5}$ have focused on $H_{a}$, and the magnetic structures have largely been resolved for this case. ${ }^{5,6}$ However, the field-induced HF phase $\left(H_{c}\right)$ and the magnetic order of Dy moments and Mn spins in this phase have yet to be explored. Neutron scattering experiments in magnetic fields $H_{c}$ are therefore highly desired to resolve the complex magnetic structures in the various phases.

It has long been speculated that magnetoelastic interactions play a major role and are the key to understanding the magnetically induced ferroelectricity and the complex phase diagrams of multiferroic $R \mathrm{Mn}_{2} \mathrm{O}_{5}$ compounds. Our results provide the experimental proof of the existence of strong magnetoelastic couplings in $\mathrm{DyMn}_{2} \mathrm{O}_{5}$. The sharp lattice anomalies observed at the phase transitions induced by temperature ${ }^{4}$ or by magnetic fields (Fig. 2) reveal the intrinsic spin-lattice coupling. Any change of the magnetic order results in a visible strain on the lattice. In addition, there is a sizable coupling of the lattice to an external field within a single phase. For example, the total change of the $c$-axis length in fields up to $8 \mathrm{~T}$ is $3 \times 10^{-4}$, far larger than the magnetostriction along $a$ or $b$. This indicates a high sensitivity of the lattice with respect to $H_{c}$, although the magnetization change in this direction is the smallest one. ${ }^{7}$ The magnetic orders and the ferroelectricity in multiferroic compounds are highly correlated. The effects of magnetostriction and multiferroic coupling cannot be separated. The current results show the macroscopic strain the lattice experiences as a result of magnetic/ferroelectric phase changes and the action of an external magnetic field. Lattice anomalies across phase transitions that are associated with a major change of the ferroelectric polarization (e.g., the transition from PE to FE3 phase) could be ascribed to multiferroic coupling effects, but the magnetic order also experiences a change at this transition. The anisotropy of the magnetostriction along the different axes provides additional insight into the magnetoelastic interactions and, in the case of $\mathrm{DyMn}_{2} \mathrm{O}_{5}$, strongly suggests the dominant role of the Dy moment order at lower temperatures.

This work is supported in part by the T.L.L. Temple Foundation, the J. J. and R. Moores Endowment, and the State of Texas through the TCSUH and at LBNL by the DOE. The work at Rutgers is supported by Grant No. NSF-DMR0520471 .
${ }^{1}$ W. Prellier, M. P. Singh, and P. Murugavel, J. Phys.: Condens. Matter 17, R803 (2005).

${ }^{2}$ G. R. Blake, L. C. Chapon, P. G. Radaelli, S. Park, N. Hur, S.-W. Cheong, and J. Rodriguez-Carvajal, Phys. Rev. B 71, 214402 (2005).

${ }^{3}$ I. Kagomiya, S. Nakamura, S. Matsumoto, M. Tanaka, and K. Kohn, J. Phys. Soc. Jpn. 74, 450 (2005).

${ }^{4}$ C. R. dela Cruz, F. Yen, B. Lorenz, M. M. Gospodinov, C. W. Chu, W. Ratcliff, J. W. Lynn, S. Park, and S.-W. Cheong, Phys. Rev. B 73, 100406(R) (2006).

${ }^{5}$ W. Ratcliff II, V. Kiryukhin, M. Kenzelmann, S.-H. Lee, R. Erwin, J. Schefer, N. Hur, S. Park, and S.-W. Cheong, Phys. Rev.
B 72, 060407(R) (2005).

${ }^{6}$ D. Higashiyama, S. Miyasaka, N. Kida, T. Arima, and Y. Tokura, Phys. Rev. B 70, 174405 (2004).

${ }^{7}$ N. Hur, S. Park, P. A. Sharma, S. Guha, and S.-W. Cheong, Phys. Rev. Lett. 93, 107207 (2004).

${ }^{8}$ N. Aliouane, D. N. Argyriou, J. Strempfer, I. Zegkinoglou, S. Landsgesell, and M. v. Zimmermann, Phys. Rev. B 73, 020102(R) (2006).

${ }^{9}$ J. Hemberger, S. Lobina, H.-A. King von Nidda, N. Tristan, V. Y. Ivanov, A. A. Mukhin, A. M. Balbashov, and A. Loidl, Phys. Rev. B 70, 024414 (2004). 